\title{
THE ISOMORPHISM PROBLEM FOR A CLASS OF PARA-FREE GROUPS
}

\author{
by BENJAMIN FINE, GERHARD ROSENBERGER and MICHAEL STILLE
}

(Received 30th October 1995)

In 1962 Gilbert Baumslag introduced the class of groups $G_{i, j}$ for natural numbers $i, j$, defined by the presentations $G_{i, j}=\left\langle a, b, t ; a^{-1}=\left[b^{\prime}, a\right]\left[b^{j}, t\right]\right\rangle$. This class is of special interest since the groups are para-free, that is they share many properties with the free group $F$ of rank 2.

Magnus and Chandler in their History of Combinatorial Group Theory mention the class $G_{h, j}$ to demonstrate the difficulty of the isomorphism problem for torsion-free one-relator groups. They remark that as of 1980 there was no proof showing that any of the groups $G_{i, j}$ are non-isomorphic. S. Liriano in 1993 using representations of $G_{h j}$ into $\operatorname{PSL}\left(2, p^{k}\right), k \in \mathbb{N}$, showed that $G_{1,1}$ and $G_{30,30}$ are non-isomorphic. In this paper we extend these results to prove that the isomorphism problem for $G_{i, 1}, i \in \mathbf{N}$ is solvable, that is it can be decided algorithmically in finitely many steps whether or not an arbitrary one-relator group is isomorphic to $G_{i, 1}$. Further we show that $G_{i, 1} \not G_{1,1}$ for all $i>1$ and if $i, k$ are primes then $G_{i, 1} \cong G_{k, 1}$ if and only if $i=k$.

1991 Mathematics subject classification: Primary 20E05, 20E06, 20 E07.

\section{Introduction}

In [2] Gilbert Baumslag introduced the class of groups $G_{i, j}$ for natural numbers $i, j$, defined by the presentations

$$
G_{i, j}=<a, b, t ; a^{-1}=\left[b^{i}, a\right]\left[b^{j}, t\right]>.
$$

(The above presentation above is slightly different than Baumslag's presentation since we use $[x, y]=x y x^{-1} y^{-1}$ and he uses $\left.[x, y]=x^{-1} y^{-1} x y\right)$. This class is of special interest since the groups are para-free, that is they share many properties with the free group $F$ of rank 2 (see [1]). In particular

(1) If $\gamma_{n}\left(G_{i, j}\right)$ are the terms of the lower central series of $G_{i, j}$, then for all $n$, $G_{i, j} / \gamma_{n}\left(G_{i, j}\right) \cong F / \gamma_{n}(F)$ and further the intersection over all $n$ of the $\gamma_{n}\left(G_{i, j}\right)$ is $\{1\}$.

(2) $G_{i, j} / G_{i, j}^{\prime \prime} \cong F / F^{\prime \prime}$ where $G_{i, j}^{\prime \prime}$ respectively $F^{\prime \prime}$ represents the second derived subgroup.

The following properties are also true of the $G_{i, j}$ (see [2] and [7]):

(3) $G_{i, j}$ has a normal subgroup with an infinite cyclic quotient group 


\section{BENJAMIN FINE, GERHARD ROSENBERGER AND MICHAEL STILLE}

(4) $G_{i, j}$ is 2 -free but not free

(5) $G_{i, j}$ is residually torsion-free nilpotent

(6) $G_{i, j}$ is hyperbolic in the sense of Gromov.

Magnus and Chandler [3] in their History of Combinatorial Group Theory mention the class $G_{i, j}$ to demonstrate the difficulty of the isomorphism problem for torsionfree one-relator groups. They remark that as of 1980 there was no proof showing that any of the groups $G_{i, j}$ are non-isomorphic. S. Liriano [7] used representations of $G_{i, j}$ into PSL $\left(2, p^{k}\right), k \in \mathbb{N}$, to show that $G_{1,1}$ and $G_{30,30}$ are non-isomorphic. In this note we extend these results on $G_{i, j}$ somewhat, by using Nielsen cancellation methods together with the techniques developed in [5] to prove that the isomorphism problem for $G_{i, 1}, i \in \mathbb{N}$, is solvable, that is it can be decided algorithmically in finitely many steps whether or not an arbitrary one-relator group is isomorphic to $G_{i, 1}$. Further we show that $G_{i, 1} \not G_{1,1}$ for all $i>1$ and if $i, k$ are primes then $G_{i, 1} \cong G_{k, 1}$ if and only if $i=k$.

\section{Preliminaries}

Notice first that $G_{i, j}$ can be expressed as an $H N N$ group

$$
G_{i, j}=\left\langle a, b, t ; t^{-1} a\left[b^{i}, a\right] b^{j} t=b^{j}\right\rangle .
$$

If in addition $j=1$ then $\langle b\rangle$ and $\left\langle a\left[b^{i}, a\right] b\right\rangle$ are maximal cyclic in $\langle a, b ;\rangle$. In $[4,5]$ techniques were developed to handle exactly such types of HNN constructions. Using the extension of Nielsen cancellation methods to HNN groups developed by Peczynski and Reiwer [10] it was proved in [4]:

Theorem A. Let $F$ be a free group and $\langle U\rangle,\langle V\rangle$ maximal cyclic subgroups of $F$. Then any two-generator subgroup of the HNN group $K=\left\langle F, t ; t^{-1} U t=V\right\rangle$ is either free, abelian or a Klein bottle group $\left\langle x, y ; x^{-1} y x=y^{-1}\right\rangle$.

Further results on the three-generator subgroups of HNN groups of the form of Theorem A were obtained in [5] with restrictions on the subgroup generated by $U, V$. A two-generator subgroup $N=<x, y>$ of a group $H$ is maximal if it is not a proper subgroup of any other two-generator subgroup of $H$; it is strongly maximal if it is maximal and for each $g \in H$ there is an $h \in H$ such that $\left\langle x, g y g^{-1}\right\rangle \subset\left\langle x, h y h^{-1}\right\rangle$ and $\left\langle x, h y h^{-1}\right\rangle$ is a maximal two-generator subgroup of $H$. Recall also that a group $G$ is $n$-free if every $n$-generator subgroup must be free. From [5] we then have the following which is a summary of several results in that paper.

Theorem B. Let $K$ be as in Theorem $A$ and suppose further that $U$ is not conjugate in $F$ to either $V$ or $V^{-1}$. Let $H=\left\langle x_{1}, x_{2}, x_{3}\right\rangle \subset K$. Then $H$ is a free group of rank at most 3 or $H$ has a presentation with one defining relation for the generating system $\left\{x_{1}, x_{2}, x_{3}\right\}$. If in addition $\langle U, V>$ is a strongly maximal subgroup of $F$ then $K$ is 3-free. 
A careful analysis of the proofs of the results in Theorem B leads us the analysis of the given class of para-free groups. We first review some of the ideas on Nielsen reduction in HNN groups. Nielsen cancellation methods are in general a very powerful tool for dealing with infinite groups. Nielsen theory was extended to free products with amalgamation by Zieschang [15] and then refined a bit by Rosenberger [12,13] and Kalia and Rosenberger [6]. It was carried over to HNN groups by Peczynski and Reiwer [10].

Let

$$
K=\left\langle B, t ; r e l . B, t^{-1} K_{1} t=K_{-1}>\right.
$$

be an HNN group with base group $B$, stable letter or free part $t$ and associated subgroups $K_{1}, K_{-1}$ (see [8] for additional information on the HNN construction). For an element $x \in K$ a representation

$$
x=h_{1} t^{t_{1}} h_{2} t^{t_{2}} \ldots h_{n} t^{t_{n}} h_{n+1}
$$

with $\epsilon_{i}= \pm 1, h_{i} \in B$ is said to be reduced if $\epsilon_{i+1}=-\epsilon_{i}$ implies that $h_{i+1} \notin K_{\epsilon_{i+1}}$.

Choose left transversals $R_{1}$ of $K_{1}$ and $R_{-1}$ of $K_{-1}$ in $B$ where $K_{1}$ and $K_{-1}$ are represented by 1 . Then each $x \in K$ may be uniquely represented as

$$
x=l_{1} t^{t_{1}} l_{2} t^{t_{2}} \ldots l_{n} t^{t_{n}} b
$$

with $\epsilon_{i}= \pm 1, b \in B, l_{i} \in R_{\epsilon_{i}}$ and $\epsilon_{i}=\epsilon_{i+1}$ whenever $l_{i+1}=1$. The length $L(x)$ of $x$ is then defined to be $n$.

As right transversals of $K_{1}, K_{-1}$, we take the inverses $R_{1}^{-1}, R_{-1}^{-1}$. Then each $x \in K$ has a reduced representation

$$
x=l_{1} t^{t_{1}} \ldots l_{m} t^{t_{m}} k_{x} t^{v_{m}} r_{m} \ldots t^{v_{1}} r_{1}
$$

with $m \geq 0, \epsilon_{i}, v_{i} \in\{ \pm 1\}, l_{i} \in R_{i}, r_{i} \in R_{-v_{1}}^{-1}$ and $k_{x}=h_{1} t^{t} h_{2}, h_{1}, h_{2} \in B, \epsilon= \pm 1$, if $L(x)$ is odd or $k_{x} \in B$ if $L(x)$ is even. In this representation $l_{1} t^{t_{1}} \ldots l_{m} t^{t_{m}}$ is called the leading half, $t^{\prime m} r_{m} \ldots t^{\prime \prime} r_{1}$ the rear half and $k_{x}$ the kernel of $x$ respectively. The above reduced representation is then called a symmetric form for $x$.

We now introduce an ordering on $K$. We assume that the groups are countable. This is convenient and no restriction if one considers a given finite generating system in $K$, for given a finite system a suitable order can always be chosen so that this system can be carried by a Nielsen transformation in finitely many steps into what we will call a Nielsen reduced system. Choose a total order of the transversals $R_{1}, R_{-1}$, and order products $l_{1} t^{t_{1}} \ldots l_{m} t^{t_{m}}$ by using the lexicographic order on the sequences $\left(l_{1}, \ldots, l_{m}\right)$. Next we extend this order to the set of pairs $\left\{g, g^{-1}\right\}, g \in K$, where the notation is chosen such that the leading half of $g$ precedes that of $g^{-1}$ with respect to the above ordering. Let $\left\{g, g^{-1}\right\}<\left\{h, h^{-1}\right\}$ if either $L(g)<L(h)$ or $L(g)=L(h)$ and the leading half of $g$ strictly precedes that of $h$ or $L(g)=L(h)$ and the leading halves of $g$ and $h$ coincide while the leading half of $g^{-1}$ precedes that of $h^{-1}$. Hence if $\left\{g, g^{-1}\right\}<\left\{h, h^{-1}\right\}$ and $\left\{h, h^{-1}\right\}<\left\{g, g^{-1}\right\}$ then at most the kernels of $g$ and $h$ may be different.

For $g \in K$ let the leading half of $g^{d(\theta)}, \epsilon(g)= \pm 1$, precede that of $g^{-\epsilon(\theta)}$. 


\section{BENJAMIN FINE, GERHARD ROSENBERGER AND MICHAEL STILLE}

A finite system $\left\{g_{1}, \ldots, g_{m}\right\}$ in $K$ is called shorter than a system $\left\{h_{1}, \ldots, h_{m}\right\}$ if $\left\{g_{i}^{c\left(g_{i}\right)}, g_{i}^{-\epsilon\left(g_{i}\right)}\right\}<\left\{h_{i}^{\epsilon\left(h_{i}\right)}, h_{i}^{-\epsilon\left(h_{i}\right)}\right\}$ holds for all $i \in\{1, \ldots, m\}$ and at least for one $i \in\{1, \ldots, m\}$, $\left\{h_{i}^{e\left(h_{i}\right)}, h_{i}^{-c\left(h_{i}\right)}\right\}<\left\{g_{i}^{e\left(g_{i}\right)}, g_{i}^{-c\left(g_{i}\right)}\right\}$ fails to hold.

A system $\left\{g_{1}, \ldots, g_{m}\right\}$ in $K$ is said to be Nielsen reduced or minimal with respect to $<$ if either $\left\{g_{1}, \ldots, g_{m}\right\}$ cannot be carried into a system $\left\{h_{1}, \ldots, h_{m}\right\}$ with $h_{i}=1$ for some $i \in\{1, \ldots, m\}$ or there is no system Nielsen equivalent to $\left\{g_{1}, \ldots, g_{m}\right\}$ which is shorter. If the group $K$ is countable, then each finite system, as in the case of a free product with amalgamation, can be carried by a Nielsen transformation into a minimal system. In general for a given finite system a suitable order can always be chosen so that this finite system can be carried by a Nielsen transformation into a minimal system.

The following is a slightly refined summary of the main results of Peczynski and Reiwer [10].

Theorem C. [10] Let $K=\left\langle t, B ;\right.$ rel.B, $t^{-1} K_{1} t=K_{-1}>$ be an HNN group. If $\left\{x_{1}, \ldots, x_{m}\right\}$ is a finite system of elements in $K$, then there is a Nielsen transformation from $\left\{x_{1}, \ldots, x_{m}\right\}$ to a system $\left\{y_{1}, \ldots, y_{m}\right\}$ for which one of the following cases holds:

(i) $y_{i}=1$ for some $i \in\{1, \ldots, m\}$

(ii) Each $w \in<y_{1}, \ldots, y_{m}>$ can be written as

$$
w=\prod_{i=1}^{q} y_{v_{i}}^{f_{1}}, \epsilon_{i}= \pm 1, \text { and } \epsilon_{i}=\epsilon_{i+1}
$$

if $v_{i}=v_{i+1}$ with $L\left(y_{v_{i}}\right) \leq L(w)$ for $i=1, \ldots, q$

(iii) Some subgroup of $B$ contains $p, p \geq 1$ of the $y_{i}$ and some product of these $y_{i}$ is conjugate to a non-trivial element of $K_{1}$.

The Nielsen transformation can be chosen in finitely many steps such that $\left\{y_{1}, \ldots, y_{m}\right\}$ is shorter than $\left\{x_{1}, \ldots, x_{m}\right\}$ or the lengths of the elements of $\left\{x_{1}, \ldots, x_{m}\right\}$ are preserved.

In addition to general Nielsen cancellation in HNN groups, important also to us is the concept of an $r$-stable Nielsen equivalence class. Let $F=\left\langle a_{1}, \ldots, a_{n} ;\right\rangle, n \geq 2$, be the free group of rank $n$ with basis $\left\{a_{1}, \ldots, a_{n}\right\}$. Let $r \in F, r \neq 1$, be a freely reduced word and suppose that there is no Nielsen transformation from $\left\{a_{1}, \ldots, a_{n}\right\}$ to a system $\left\{b_{1}, \ldots, b_{n}\right\}$ with $r \in\left\langle b_{1}, \ldots, b_{n-1}\right\rangle$. We consider generating systems $\left\{r, x_{1}, \ldots, x_{n}\right\}$ of $F$ and we say that in a Nielsen transformation $\phi$ from $\left\{r, x_{1}, \ldots, x_{n}\right\}$ to a system $\left\{r, y_{1}, \ldots, y_{n}\right\}, r$ is not replaced if in all the elementary Nielsen transformations comprising $\phi, r$ either remains unchanged, is replaced by $r^{-1}$ or is put in a different place in the relevant $(n+1)$-tuple. We then refer to a Nielsen transformation and the corresponding Nielsen equivalence class in which $r$ is not replaced as $r$ stable. 


\section{Baumslag's para-free groups}

We now consider the class of para-free groups introduced by Baumslag [2]. From the introduction these have the presentations

$$
G_{i, j}=\left\langle a, b, t ; a^{-1}=\left[b^{i}, a\right]\left[b^{j}, t\right]>\right.
$$

where $i, j \in \mathbb{N}$. These groups share many properties with the free groups of rank 2 (see the Introduction). As remarked in the Introduction, Magnus and Chandler [3] in their History of Combinatorial Group Theory mention the class $G_{i, j}$ to demonstrate the difficulty of the isomorphism problem for torsion-free one-relator groups. They note that as of 1980 there was no proof showing that any of the groups $G_{i, j}$ are nonisomorphic. S. Liriano [7] used representations of $G_{i, j}$ into $\operatorname{PSL}\left(2, p^{k}\right), k \in \mathbb{N}$, to show that $G_{1,1}$ and $G_{30,30}$ are non-isomorphic. Our main result is the following.

Theorem 1. Let $i$ be a natural number. Then:

(1) the isomorphism problem for $G_{l, 1}$ is solvable, that is it can be decided algorithmically in finitely many steps whether or not an arbitrary one-relator group is isomorphic to $G_{i, 1}$;

(2) $G_{i, 1}$ is not isomorphic to $G_{1,1}$ for $i \geq 2$;

(3) if $i, k$ are primes then

$$
G_{i, 1} \cong G_{k, 1} \text { if and only if } i=k \text {; }
$$

(4) for all natural numbers $i, G_{i, 1}$ is Hopfian, every automorphism of $G_{i, 1}$ is induced by an automorphism of the free group $F^{*}=F^{*}(A, B, T)$ of rank 3, with respect to the epimorphism $A \rightarrow a, B \rightarrow b, T \rightarrow t$, and the automorphism group $A u t G_{i, 1}$ is finitely generated.

Proof. Suppose that $G$ is an arbitrary one-relator group. If $G \cong G_{i, 1}, i \geq 1$, then $G$ must have rank 3. Any isomorphism then leads to a generating system $\left\{x_{1}, x_{2}, x_{3}\right\}$ for $G_{i, 1}$. We then use the Nielsen cancellation method on this generating system to describe, up to conjugation and certain algorithmically performed substitutions, the Nielsen classes. This coupled with the Whitehead algorithm (which allows us to determine when two elements of a free group are congruent via an automorphism) and the solution of the conjugacy problem in free groups leads to the given solution of the isomorphism problem.

To apply the Nielsen techniques consider the presentation (1) and notice that in general $G_{i, j}$ can be expressed as an HNN group

$$
G_{i, j}=\left\langle a, b, t ; t^{-1} a\left[b^{i}, a\right] b^{j} t=b^{j}\right\rangle .
$$

If $j=1$ then $G_{i, 1}$ has the presentation

$$
G_{i, 1}=<a, b, t ; a^{-1}=\left[b^{i}, a\right][b, t]>
$$




\section{BENJAMIN FINE, GERHARD ROSENBERGER AND MICHAEL STILLE}

and in this case the associated subgroups $\langle b\rangle$ and $\left\langle a\left[b^{i}, a\right] b\right\rangle$ are maximal cyclic and thus malnormal in $\langle a, b$; $\rangle$. Therefore we may apply the Nielsen techniques developed in [5]. We use the notation $F=\langle a, b ;\rangle, c=a\left[b^{i}, a\right] b, d=c b^{-1}=a b^{i} a b^{-i} a^{-1}$, and recall that in the free group $F$ the conjugacy problem is solvable. Further recall that the Whitehead algorithm (see [8]) allows us to determine when two elements (or more generally two finite subsets) of a free group are congruent via an automorphism.

Now let $\left\{x_{1}, x_{2}, x_{3}\right\}$ be a generating system for $G_{i, 1}$. From the results in [5] there is a one-relator presentation for $G_{i, 1}$ on $\left\{x_{1}, x_{2}, x_{3}\right\}$. By analyzing the techniques developed in [5] (the proof of Theorem 1 together with Proposition 1 of that paper) and after replacing $t^{-1}$ by $h t^{-1}$ (or $t^{-1} h$ ) and $b$ by $h b h^{-1}$ (respectively $c$ by $h^{-1} c h$ ), $h \in F$, if necessary, we need only consider the cases

(1) $x_{1}, x_{2} \in F, c^{\alpha} \in\left\langle x_{1}, x_{2}\right\rangle, \alpha \geq 1$, and $x_{3}=t$ or

(2) $x_{1}, x_{2} \in F, x_{2}=b^{\alpha}, \alpha \geq 1$, and $x_{3}=t$.

We consider first case (1). The Nielsen cancellation method in free groups easily gives that $c=a\left[b^{i}, a\right] b$ is primitive in each proper two-generator subgroup of $F$ which contains $c$. Hence by a result of Rosenberger (Lemma 2.1 of [14]) one necessarily has $\left\langle x_{1}, x_{2}\right\rangle=F$, and $\left\{x_{1}, x_{2}, t\right\}$ is Nielsen equivalent to $\{a, b, t\}$ or some $c^{\gamma}, \gamma \geq 1$ is primitive in $\left\langle x_{1}, x_{2}\right\rangle$. If this latter case holds then we may assume that already $x_{2}=c^{y}$ which reduces the problem to case (2) after a suitable conjugation in $G_{i, 1}$ and again applying Nielsen reduction. Hence we now consider case (2).

Let $x_{1}, x_{2} \in F, x_{2}=b^{\alpha}$ for some $\alpha \geq 1$ and $x_{3}=t$. By cancellation methods in HNN groups we have necessarily that $F=\left\langle x_{1}, x_{2}, c^{\alpha}\right\rangle=\left\langle x_{1}, b^{\alpha}, c^{\alpha}\right\rangle$ independently of whether $c \in\left\langle x_{1}, x_{2}\right\rangle$ already or not. Further we must then have $\alpha=1$ since the factor group $\bar{F}=\left\langle a, b ; b^{\gamma}=\left(a\left[b^{i}, a\right] b\right)^{\gamma}=1\right\rangle, \gamma \geq 2$, is not cyclic (see [4]). Therefore we have $F=\left\langle x_{1}, b, c\right\rangle=\left\langle x_{1}, b, d\right\rangle$. We wish to show that, in a minimizing manner, $\left\{x_{1}, b, c\right\}$ is c-stable (see previous section) Nielsen equivalent to $\{a, b, c\}$, and hence $\{x, b, t\}$ is Nielsen equivalent to $\{a, b, t\}$, or if $i \geq 2,\left\{x_{1}, b, c\right\}$ is c-stable Nielsen equivalent to a system $\left\{b, a b^{\beta} a^{-1}, c\right\}$, and hence $\left\{x_{1}, b, t\right\}$ is Nielsen equivalent to $\left\{b, a b^{\beta} a^{-1}, t\right\}$, where $1 \leq \beta<i, \beta \mid i$.

We consider the generating system $\left\{x_{1}, b, c\right\}$ and perform c-stable Nielsen transformations from $\left\{x_{1}, b, c\right\}$ to other systems in a minimizing manner. For this we regard $F$ as the free product $F=\langle a\rangle *\langle b\rangle$ of two infinite cyclic groups together with the length $L$ and an order with respect to this factorization. The reason for doing this is that in this factorization $L(c)=6$ whereas the free length of $c$ is $2 i+4$ which can be quite large. We note that $H=\langle b, c\rangle=\langle b, d\rangle$ is free of rank 2 , and there is no cancellation between $b$ and $d=a b^{i} a b^{-i} a^{-1}$ with respect to the above free product factorization. In particular we have that $H \varsubsetneqq F$. We write $x=x_{1}$ and now use the Nielsen cancellation method in the free product factorization for $F$ to handle $\{x, b, c\}$. For each $\mu, \epsilon= \pm 1$ and for each $r \in H$ we can obtain

$$
\begin{gathered}
L\left(x^{\mu} r^{\prime}\right) \geq L(x) \text { and } \\
L\left(x^{\mu} r x^{\epsilon}\right)>L(x)-L(r)+L(x) .
\end{gathered}
$$


In particular the reduced form of $x$ does not end with a power of $b$.

Consider first the case where $L(x) \geq 5$. Since $F=\langle x, b, d\rangle$ we must have either at least one $L\left(x^{\mu} d^{\epsilon}\right)<L(d)=5$ or always $L\left(x^{\mu} d^{k}\right) \geq L(d)=5$, but at least once $L\left(d^{\mu} x d^{d}\right) \leq$ $2 L(d)-L(x)=10-L(x), \mu, \epsilon= \pm 1$. In the latter situation the left half and the right half of the symmetric normal form of $x$ are cancelled whence we obtain $L\left(x^{y} d^{\beta}\right)<L(x)$ for some $\beta \neq 0, \gamma= \pm 1$, which contradicts $L\left(x^{\mu} r^{\epsilon}\right) \geq L(x) ; \mu, \epsilon= \pm 1 ; r \in H$. Therefore we have $L\left(x^{\mu} d^{\epsilon}\right)<5$ for some $\mu, \epsilon= \pm 1$ and we may replace $x$ by $y=x^{\mu} d^{\epsilon}$. Hence from the beginning we may assume that $L(x)<5$.

Therefore we now consider the case where $L(x)<5$. Since $x$ neither starts nor ends with a power of $b$, we must have $L(x)=1$ or $L(x)=3$. Consider first $L(x)=3$. In this case then $x=a^{\alpha} b^{\beta} a^{y}$ for some non-zero integers $\alpha, \beta, \gamma$. Assume that $L\left(x^{\mu} d^{\epsilon}\right)<L(d)=5$ for some $\mu, \epsilon= \pm 1$. Suppose for example $L(x d)<5$. Then we have necessarily $\gamma=-1, \beta=-i$ and $x d^{-\alpha}=a^{\alpha} b^{-i} a^{-1} a b^{i} a^{-\alpha} b^{-i} a^{-1}=b^{-i} a^{-1}$, that is $L\left(x d^{-\alpha}\right)<L(x)$ which contradicts that $L\left(x^{\mu} r^{\epsilon}\right) \geq L(x) ; \mu, \epsilon= \pm 1 ; r \in H$. The same type of analysis works for the other possibilities for $\mu, \epsilon$ and hence we always have $L\left(x^{\mu} d^{\epsilon}\right) \geq L(d)$. We must have at least once $L\left(d^{\mu} x d^{c}\right) \leq 2 L(d)-L(x)=7$ and then we have necessarily that $\alpha=1$ and $\gamma=-1$. This implies then that $x=a b^{\beta} a^{-1}$. Without loss of generality let $\beta \geq 1$. We must have $1 \leq \beta \leq i, \beta \mid i$ because for $\beta \geq 2, \beta \chi i$, the factor group $\bar{F}=\left\langle a, b ; b^{\beta}=1\right\rangle$ cannot be generated by $b$ and $a b^{i} a b^{-i} a^{-1}$. Let $x=a b^{\beta} a^{-1}$ with $1 \leq \beta \leq i, \quad \beta \mid i$, and $j=\frac{i}{\beta}$. We then have the relation $a=x^{-j} d x^{j}$ and hence $G_{i, 1}=\left\langle x, b, t>\right.$. If $\beta=i$ then we have $x^{-1} d=a^{2} b^{-i} a^{-1}$ and $L\left(x^{-1} d\right)=3<L(d)=5$ which contradicts that $L\left(x^{\mu} d^{c}\right) \geq L(d), \mu, \epsilon= \pm 1$, and therefore we may assume that $\beta<i$.

Hence let $i \geq 2$ and $\beta<i$. Here $\{x, b, c\}$ is c-stable Nielsen equivalent, in a minimizing manner, to $\left\{b, a b^{\beta} a^{-1}, c\right\}$ and hence $\{x, b, t\}$ is Nielsen equivalent to $\left\{b, a b^{\beta} a^{-1}, t\right\}$. Using the relation $a=x^{-j} d x^{j}=x^{-j} t b t^{-1} b^{-1} x^{j}$, where $x=a b^{\beta} a^{-1}$ we get for this generating system $\{x, b, t\}, x=a b^{\beta} a^{-1}$, the one-relator presentation

$$
G_{i, 1}=\langle x, b, t ; R=1\rangle
$$

where $R=x^{-j} b t b^{-1} t^{-1} x t b t^{-1} b^{-1} x^{j} b^{-\beta}$ and $i \geq 2,2 \leq j \leq i, j \mid i$ and $\beta=\frac{1}{j}$. This completes the case when $L(x)=3$.

Now suppose $L(x)=1$, that is, $x=a^{\alpha}$ for some $\alpha \neq 0$. We may assume that $\alpha \geq 1$; but then we must have $\alpha=1$, for if $\alpha \geq 2$ the factor group $\bar{F}=<a, b ; a^{\alpha}=1>$ cannot be generated by $b$ and $a b^{i} a b^{-i} a^{-1}$. Therefore here $\{x, b, c\}$ is, in a minimizing manner, $c$-stable Nielsen equivalent to $\{a, b, c\}$ and hence, $\{x, b, t\}$ is Nielsen equivalent to $\{a, b, t\}$. We note also that this always holds if $i=1$, that is in particular we have: If $\left\{x_{1}, x_{2}, x_{3}\right\}$ is a generating system for $G_{1,1}$ then $\left\{x_{1}, x_{2}, x_{3}\right\}$ is Nielsen equivalent to $\{a, b, t\}$. In all cases the Whitehead method now leads to the solvability of the isomorphism problem for the class $G_{i, 1}, i \in \mathbb{N}$. In particular it shows that $G_{1,1}$ is never isomorphic to $G_{i, 1}$ with $i \geq 2$ because the latter group always has at least two different Nielsen equivalence classes of generating triples while $G_{1,1}$ has exactly one Nielsen equivalence class of generating triples. By examining the effect of Whitehead moves in the rank 3 free group $F$ with ordered basis $\{X, Y, Z\}$ on the words $X\left[Y^{i}, X\right][Y, Z], i \in \mathbb{N}$ 


\section{BENJAMIN FINE, GERHARD ROSENBERGER AND MICHAEL STILLE}

and $X^{-j} Y Z Y^{-1} Z^{-1} X Z Y Z^{-1} Y^{-1} X^{j} Y^{-\beta}, i \geq 2,2 \leq j \leq i, j \mid i$ and $\beta=\frac{1}{j}$, we see that they have minimal free length among all words which we can obtain from them via automorphisms of the free group $F$. Further for different $i$ and $k$ these corresponding words have pairwise different length. Therefore the above also shows that if $i, k$ are distinct primes then $G_{i, 1} \not G_{k, 1}$.

The remaining statements in the theorem are now almost immediate. Let $\phi: G_{i, 1} \rightarrow G_{i, 1}$ be a homomorphism from $G_{i, 1}$ onto $G_{i, 1}$, and set $a^{\prime}=\phi(a), b^{\prime}=\phi(b), t^{\prime}=\phi(t)$. Consider the generating system $\left\{a^{\prime}, b^{\prime}, t^{\prime}\right\}$. Applying Nielsen transformations to this system with respect to the equation $t^{\prime-1} a^{\prime}\left[b^{i}, a^{\prime}\right] b^{\prime} t^{\prime}=b^{\prime}$ shows that $\left\{a^{\prime}, b^{\prime}, t^{\prime}\right\}$ is Nielsen equivalent to $\{a, b, t\}$ and hence $G_{i, 1}$ is Hopfian. Further every automorphism of $G_{i, 1}$ is induced by an automorphism of the rank 3 free group. Since the group of inner automorphisms is finitely generated it follows that Aut $G_{i, 1}$ is finitely generated (see [9]). This completes the theorem.

\section{REFERENCES}

1. G. BaumslaG, A survey of groups with a single defining relation, in Proceedings of Groups St Andrews 1985 (1986), 30-58.

2. G. Baumslag, Groups with the same lower central series as a relatively free group. II. Properties, Trans. Amer. Math. Soc. 142 (1969), 507-538.

3. B. Chandler and W. MaGnus, The History of Combinatorial Group Theory (SpringerVerlag, 1982).

4. B. Fine, F. ROHL and G. ROSENBERGER, Two generator subgroups of certain HNN groups, Contemp. Math. 109 (1990), 19-23.

5. B. FINE, F. ROHL and G. ROSENBERGER, On HNN groups whose three-generator subgroups are free, in Infinite Groups and Group Rings (World Scientific, 1993), 13-37.

6. R. N. KALIA and G. ROSENBERGER, Uber Untergruppen ebener discontinuelicher Gruppen, Contemp. Math. 33 (1984), 308-327.

7. S. LIRIANo, The non-isomorphism of two one-relator groups is established (Ph.D. thesis, New York 1993).

8. R. C. LyNdon and P. E. SchUPP, Combinatorial Group Theory (Springer-Verlag 1977).

9. J. McCool, Some finitely presented subgroups of the automorphism group of a free group, J. Algebra 35 (1975), 205-273.

10. N. Peczynski and W. Rerwer, On cancellations in HNN groups, Math. Z. 158 (1978), 79-86.

11. N. Peczynski, G. Rosenberger and H. Zieschang, Uber Erzeugende ebener diskontinuierlicher Gruppen, Invent. Math. 29 (1975), 161-180. $1-12$.

12. G. Rosenberger, Gleichungen in freien Produkten mit Amalgam, Math. Z. 173 (1980),

13. G. Rosenberger, Zum Rang und Isomorphieproblem fur freie Produkte mit Amalgam (Habilitationsschrift, Hamburg 1974). 
14. G. RosenBerger, On one-relator groups that are free products of two free groups with cyclic amalgamation, in Groups St Andrews 1981 (1983), 328-344.

15. H. ZIEschang, Uber die Nielsensche Kurzungsmethode in freien Produkten mit Amalgam, Invent. Math. 10 (1970), 4-37.

Department OF Mathematics

FAIRFIELD UNIVERSITY

FAIRFIELD, CONNECTICUT 06430

U.S.A.

FaChbereich Mathematik Universitat

DORTMUND

44221 DORTMUND

Federal Republic of Germany
FaChbereich Mathematik Universitat

DORTMUND

44221 DORTMUND

Federal Republic of Germany 